\title{
Squaring the Dialectic of Inference and Chance
}

\author{
Paul Healey \\ Independent Scholar
}

\begin{abstract}
Instead of merely inferring that our beliefs are in a correlation to the facts, I will contend that what matters is the logic of their inferential structure. This will reveal what is actually true for their relation and not just real for experience. Given one and two-sided inferences, the conditions of their functional relation will be consistent within a square of opposition; for contraries, contradictions and sub-contraries in a way that probability theory is not. Within this model of inferences, the two-sided concept of inferences will also have a modal value for its relation to the one-sided concept of inferences. Proposing that empirical, formal, speculative, and an Intuitionist Speculative understanding of logic will have a modal identity within a dialectical principle.
\end{abstract}

Keywords: inference, square of opposition, chance, modalities, dialectic, principle

\section{Introduction}

In Section 1, I will make relevant my interpretation of Aristotle's work on oppositions. This is such an analysis of Aristotle's (Metaphysics, 9.2) concept of inference which can be interpreted as a two or one-way capacity $^{1}$ to process experiences. From Section 2, it will follow the empirical and its formal justification of probability theory can be falsified. Truth according to the postulates of its definitions will not be what is true for chances. Both ways of thinking will be further examined in Section 3 by considering their relation as methods for making predication. Once this is done, in Section 4, the model of inferences will be set for oppositions on the basis of the theorems proposed for different understandings of logic. After that I will update the principle of the dialectic (the author) in the conclusion to take into account the concept of their modal demarcation for the model of inferences.

\subsection{Inferring the Oppositions of a Proposition}

It seems much easier to explain why a square of opposition (Be'ziau 2012) is useful for comprehending inferences than it is for demonstrating them. Consider an opposition where it seems fairly easy to decide which propositional form is true. I.e., Einstein's theory of special relativity (Bohm 1965) will always be an advance on Newton's theory of mechanics. This contradicts that it might not always be so, or is contrary to that it is never the case that Einstein's special relativity is an advance on Newton's mechanics. When it is never the case, this

Paul Healey, Independent scholar, UK; main research fields: Philosophy of Dialectic, Logic, Maths, and Science. Email: paulmsrf@btinternet.com.

Acknowledgments: To Walter Felscher, I am indebted for the interest he showed in the opposition between two simple rules for binary sequences that appear to be random, but are not. Also I am indebted to Roger Bishop Jones for acknowledging that my notion of effective calculability is different from Alonzo Church's, but needed more work; to show the computability of a function isn't necessarily synonymous with being an effective decision procedure. Special thanks to Martin Cohen for helping me with my first published work. These concepts also benefited from discussions as a result of Jean-Yves Be'ziau and the organizers for letting me present my work Back to Hegel and the Right Square on May 5-9th, 2014 at the Square of Opposition conference at the Pontifical Latern University. Jonathan L. Shaheen also provided some useful criticism for my 2014 article. 
contradicts it can be the case, so both sub-contraries collapse into particular alternatives. Not knowing that Einstein extended relativity to optics and electrodynamics doesn't imply that there is no knowledge of how experiences are processed. Therefore, it can easily be deduced that it is not just the senses that are necessary for thinking; it is not just an eye, nose, tongue, limb, or ear, but a brain that is required as well. Now since each brain requires a mind which further distinguishes it from other brains, many properties can be universally and particularly affirmed or denied for how it thinks. I.e., it can be universally affirmed that a brain is always required for thinking as opposed to never, sometimes or not sometimes so required. Unlike the properties of physical experiences and objects like that of tables, wheels, and fruit etc., inferences can be set out so as to explain how they are processed as thoughts by the mind.

In opposition to Maken and Denyer (2000), it can be asserted that physical objects do not just have the capacities and potentials that the mind can presuppose they have. Even though knowledge beyond the capacity of the mind is unintelligible to it, what are intelligible are the experiences of its body and they do not go beyond it. Without a disposition that has capacities, like desire, imagination, and creativity, it cannot have developed old theories so they can be replaced by new ones. Theories constituted by inferences that are presupposed to be merely intrinsic only have an antecedent and postcedent structure, whereas they can also be extrinsic given they have consequences if acted upon. How else to determine these if not by their relation? Being rational or irrational capacities must then mean the properties of inferences provide reasons for the advance of their analysis. With two types of inference, one-sided $I 1$ and two-sided $I 2$, their relation for a square of opposition can be set out as necessary $\square$, possible $\diamond$, impossible $\neg \diamond$, and contingent $\nabla$ :

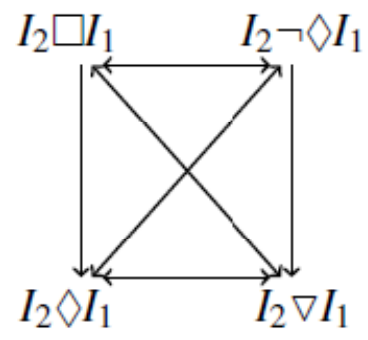

1. Modal inference of oppositions

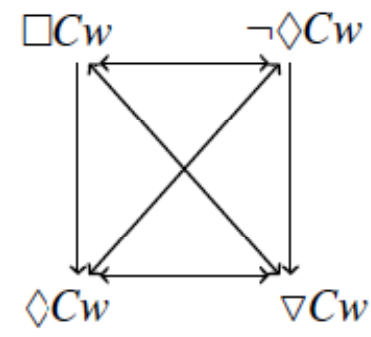

2. Counterpart worlds of opposition

For figure 1 and figure 2, the diagonals are the contradictions; the top horizontal represents the contraries and the lower the sub-contraries, while the vertical down arrows are what is entailed from the universal to the particular forms of modal relations. Unlike figure 1, the reasoning for figure 2 is to make the modalities hold for the counterpart $C$ being a property of the world $w$, but as it is hoped, it will become clearer, the contradictories for the $C$ property have no resolution given the relation of $I 2$ to $I 1$ is not admissible.

\subsection{A Contradictory Correlation}

Natural, realistic and even transcendental ways of thinking about language tend to sever real experience from the actual oppositions that hold for the understandings of its forms. To many following Russell (1931), who fails to get the significance of Leibniz' contradiction... "the one-one correlation of whole and part, which underlies Achilles" (355), Hegel's Science of Logic (Giovanni 2010) fails to provide reasons why experiences can be more or less intelligible. They can only be so for the dialectic given a unity of oppositions supersedes any formal analysis dependent on propositional functions. 
While inferences can be rational or irrational for affirming propositions, this is not so for the one-way capacity of cognition. Experiences are not just random in that none of their initial conditions are known or ordered. Rather the state of affairs can be more or less complex for them. Taking $X$ to be random so $\operatorname{pr}(X=x)$ (Kimbrough 1980) to predict the successes $x$ for any quantity of trials $X$, presupposes $x$ is an independent variable. It might therefore be tempting to deduce that dependent variables belong to formulas that are purely mechanical or physical for the states of properties. The problem with this is that the forms of such formula belong to functions which are not independent of chance. More is needed to prove that the differences for the two types of variable are relevant. If inferences can be in a relation of oppositions, they can be substantiated by functions that calculate their differences and ones that can't. Being so, dialectical analysis should constitute their relation if there is to be an objective science of logic. What is actually the case for the relation of inferences is then so for the experience of events.

\subsection{Proportions in Their Correspondence to Distributions}

Thoughts thinking about other thoughts can after all be in an opposition to them. Given the additive and multiplicative laws are ones which can be empirically understood (Lewis 1980), it might seem that there is no logic to account for the difference and reasoning of inferences. Even though the addition of the hypothesis has a sum that need not be 1, the probabilities of hypotheses mean the likelihood function: $L(H \mid E)=d f P\{E \mid H\}$ (Kimbrough 1980) just defines the likely hypothesis to be the one where the events are the most probable and not how to predict or select them. What it does not take into account is that limiting likelihood to the one-way capacity of independent variables might mean that its function cannot be a rational method for selecting proportions. ${ }^{2}$ These if I am right can also be improbable given it is not necessary that they are independently distributed. Neither do the formulas for measures of explanatory hypothesis' considered by Glass (2014) acknowledge that proportions can consist of independent or dependent variables. Actual distributions are not necessarily ordered according to the possible combinations of a proportion, but can just as easily be logistically ordered for possible distributions. E.g., taking the subset of the ordering for the proportion of a $1 / 2$, you get the combinations for three possible outcomes:

$$
\{0,0,0\},\{0,0,1\},\{0,1,0\},\{0,1,1\},\{1,0,0\},\{1,0,1\},\{1,1,0\},\{1,1,1\}
$$

where there are three possible sets with 1 unit and two zeros belonging to them, you get a probability of $3 / 8$, but allowing for its negation; $1-3 / 8$ to get the improbability of $5 / 8$, the possibility of one unit and two zeros need not be independent and combinatorially ordered. The same reasoning holds for other proportions like $1 / 3$ or any other rationals between zero and 1 .

Empirical alternatives where the choice of three outcomes do not have the same quantity of units and or zeros presuppose independence, so dependence cannot be differentially inferred from the same function. Since, the improbabilities of their proportion could in fact have a greater quantity of successful predictions for other distributions. Given the proportion of the hypothesis is in error, limiting them to the probabilities of the hypotheses that have the most success is no guarantee of future successes. Any correlation with its degree of belief cannot therefore count as evidence for its objectivity. The empirical evidence of probable correspondences of proportions to their possible distributions therefore relies on indeterminate correlations and not the proportion of the variables in a union with their difference. 
Taking degrees of belief as percentages so as to combine alternative hypotheses (Lewis 1980), it might appear all other understandings of logic are easily subjugated to an empirical one. Any correlation ${ }^{3}$ of empirical judgments to experience cannot falsify them being anything other than hypothetical.

Reasoning about chance, many (Carnap 1945; Reichenbach 1949; Ayer 1957; Lenz 1973; Ackermann 1961; Barker 1957, 1961; Hillman 1962; Braithwaite 1959; Russell 1962; Lewis 1980; Popper 1959; Cohen \& Nagel 1962; Van Fraassen 1995; Ha'jek 2003; Pfiefer 2014) have justified their belief or critique of inferences that ground the logical interpretation of probability on notions such as simplicity, hypothesis, corroboration, confidence, and superiority. None though has considered the proportion as a hypothesis for the prediction of a given distribution, as anything other than probable. ${ }^{4}$ Whereas when their relation is improbable, this suggests they do not correspond. This implies the difference between being probable and improbable matters. Certainly for human decision making, even if not consciously thought about, the relation of successful to unsuccessful predictions should not be denied when it comes to recognizing a skill like being a fast swimmer, as opposed to what is required for it like the right physique. Not accounting for the relation between the two-sided and one-sided way, the frequency of a distribution's properties is weighed and seems a huge oversight.

Falsifying the relation for the types of inference would no doubt require evidence that the differences between probabilities and improbabilities do not constitute their likelihood. Grossman $(2011,576)$ attributes Bayesian Hegelianism to the fact that prior probabilities are needed to apply a Likelihood Principle. ${ }^{5}$ Taking an historical dialectic into account, even though Grossman does not spell it out; it should be clear that the likelihood is not itself a principle but rather a property required for the modality of a dialectic one. By taking the difference between probabilities and improbabilities as distinct from the dependency of empirical evidence on hypothetical thinking, the confidence interval can be significant for predicting chances; consider two postulates for rational decision making:

$$
\begin{aligned}
& \text { P1: } p r \mid i m(s \mid p \& d)=C h(s) \\
& \text { P2: } p r \mid i m(p / d)=l p
\end{aligned}
$$

For P1, the idea for the use of the stroke in $p r \mid i m$ is that the differences for the states $s$ being probable or improbable or equal can be calculated given the proportion $p$ and the distribution $d$ to get the chance $C h$ of the states $s$. P2 just allows for the fact that the proportion given a distribution can be more or less likely, but being so does not undermine the learning that it is not exclusively independent or dependent of the likelihoods proportion. Since all the variables have a reasoning for being deduced, the inference is more than just a mere hypothesis conditional for some antecedent and postcedent. For with P1 and P2, the type of inference counts as evidence for the utility of their consequences. With this the capacities of the inferences can also be in an opposition for their relation that is not reducible to their likelihood! If I have not erred, this means that predictive inferences have a structure within a system of relations; so any reduction of their concepts to their mere correlation with the system data will be a weakening of it.

What can then count as a presupposition is a theorem, where an unknown set of weighted choices belonging to a game of chance will produce a distribution that can be successfully predicted by a theory of probability (Kolmogorov 1956). Not holding for a unity of oppositions, the outcomes of the probability functions have no decision procedure for making successful predictions. Generalizing this theorem, reasons for making decisions cannot be modeled on having a skill. 
The problem with probability theory is that hypothetical proportions can in fact correlate to some degree to the distribution of a possible data set. That is, this gives the illusion that it is a basis for sound inference. Neither does corroboration of the likelihoods being coherent with Kolmogorov's theorem of total probability and Bayes' undermine any difference between the measure of probabilities and improbabilities. Given their success and errors are abducted by the presupposition of the hypothetical proportion, probability theory and its hypothetical corpus (Kyberg 1990) has no rational decision procedure. Neither is there satisfactory justification of probability theory by those who call themselves intuitionists. ${ }^{6}$ By proposing that there is a modal difference between empirical, formal, speculative, and Intuitionist Speculative understanding of logic, the relation of conditional probabilities to unconditional ones (Fitelson \& Ha'jek 2014) also avoids the structure of their relations within a square of oppositions. Given the significance of the binomial and later the normal or Gaussian distribution, the correspondence between a hypothetical proportion and the way it is distributed for events need not be presupposed.

Giving a particular example, clearly it is ill conceived to presuppose that the average debt is neatly distributed, so the extremes of not having any and being bankrupt are uniform for the history of the whole population. No doubt it is possible for some populations to have very little debt and some to have too much. The point is that there should be no expectation that the state of affairs is not dynamic for any size of population. Say the proportion of debt to earnings is $3 / 4$ and debt is represented by units of currency and zero's the privation of it, when the history of their distribution is closer to their proportion the prediction of the probabilities will have a greater success for their measure than the improbabilities; when it is otherwise the improbabilities will have a greater success, if the agent pays off more or less of the debt given by the proportion; when the improbabilities as calculated from the probabilities have a greater success of predicting the increase or decrease in debt from the history of the units and zeros distributed overrides psychological preconceptions. If not, the difference between them would not be significant. By adjusting the proportion to maximize the likelihood of predicting the next payment the significance of the calculation can also be amended. Modeling the likelihoods this way, it appears; if I have not made an error, the one-sided use of statistical inference and its hypothetical corpus to calculate probabilities can be superseded.

No arbitrary closeness of the proportion to the distribution undermines the efficacy of the chance functions likelihood, as there is no presupposition to doubt it. This counters the presumption that the payments have a fair chance of being made when they are less likely to be.

No doubt making this relevant to the philosophical understandings of inference is much harder, but I think this can be done by considering what the negation of a probability function means by extrapolating it from Hegel's (Giovanni 2010, 595) one-sided understanding of syllogistic inference; the critique is applicable to: $1-p r(p / d)=p r(p / d), 1-p r(p / d)=\neg p r(p / d)$, or $1-p r(p / d)=i m(p / d)$. Applying the two-sided understanding of its middle concept: $\operatorname{pr} \mid i m(p / d)$, the history of the states for their extremes, e.g., payments or non-payments for taking on a debt, can be predicted on the basis of a proportion which is not in a purely hypothetical correspondence of success or failure to them. That is to say, the negation of probability function is not evidence that there is no relation of difference constitutive of information. When the probabilities do correspond, they will have a greater success in predicting the outcomes of taking on the debt. Taking the difference between both sides therefore appears to warrant a greater degree of confidence. Such confidence also seems to suggest a more objective interpretation of Bayes' theorem and the multiplicative and additive rule of total probability (Kolmogorov 1956) is possible. Formal theoretical thinking can therefore be accused of asserting rules from a 
standpoint of system that is in-itself for-itself. Just denying the relation of opposition to the other, there can be no unity. Whereas empirical thinking can be accused of reducing their theoretical truth to the chance of the belief in a proposition to being true or not true for some event. Rather propositions which are closer or further from the truth have a chance of being thought or relevant, but not a chance of being closer or further from their actual truth.

Even in the more sophisticated exposition of the imprecise credence model (Joyce 2010), the reason for them being perfectly correlated, anti-correlated, or in some kind of intermediate correlation is not given. Making the Creedal state in some event say $D$, so nothing else depends on some post-learning state for some credence function Joyce gets $C D=\{\mathbf{c}(\bullet \mid D)=\mathbf{c}(X) \cdot[\mathbf{c}(C \mid X) / \mathbf{c}(D)]: \mathbf{c} \in C\}$. From this, Joyce claims the elements of the credence function $C D$ are intuitively probability functions that the believer takes to be compatible with their total evidence. The so called subjectivity of the imprecise Bayesian model fares is not better than its precise model, as the analysis does not incorporate what it means to have a degree of belief in an understanding of logic that it transcends or is transcended by. If it could than $C D$, it could simultaneously be some other degree of belief in opposition to it. Given it cannot be the credence, function cannot be the function of the dialectic, as the latter differentiates the modal value of other understandings of logic (the author), so the relations of their methods can be coherent.

Also it can easily be argued that credence is not a function of cognition, but the result of processes that weigh experiences like feelings; convictions, passions, and desires. By abstraction, denial of inferential types $C D$ becomes an empirical theory of chance where the higher or lower standpoints of system for the modal predication of other credence within it become unintelligible.

Now taking what Lewis' (1968) proposes for the modal terms for quantified modal logic as they correspond to figure 2. What he doesn't consider is its square of opposition as given by figure 1; even if it is thought that I have taken a liberty with his translation of $\mathrm{D} F x$, the property of $F$ remains no less mystical even if it is a counterpart $C$. With Lewis' predicated translation, let $L u a$ be Lewis' universal affirmation, given below for $\mathrm{D} F x$, only the probability theory allows for the faulty correspondence between hypothetical proportions and the prediction of their likely distribution; squaring to get $L p a, L u d$, and $L p d$, the universal affirmative: $L u a$ is contradicted by the particular denial $L p d$, so the truth of his counterpart theory begs his cherished idea of Humean supervenience, where the truth comes after being:

$$
\begin{aligned}
& L_{u a}: \forall y_{1} \forall x_{1}\left(W y_{1} \& I x_{1} y_{1} \& C x_{1} x \supset F x_{1}\right) \\
& \text { (Every counterpart of } \mathrm{x} \text {, in any world, is an F) } \\
& L_{p a}: \forall y_{1} \exists x_{1}\left(W y_{1} \& I x_{1} y_{1} \& C x_{1} x \supset F x_{1}\right) \\
& \text { (Some counterpart of } \mathrm{x} \text {, in any world, is an F) } \\
& L_{u d}: \forall y_{1} \forall x_{1}\left(W y_{1} \& I x_{1} y_{1} \& \neg C x_{1} x \supset F x_{1}\right) \\
& \text { (No counterpart of } \mathrm{x} \text {, in any world, is an F) } \\
& L_{p d}: \forall y_{1} \exists x_{1}\left(W y_{1} \& I x_{1} y_{1} \& C x_{1} x \supset \neg F x_{1}\right) \\
& \text { (Some counterpart of } \mathrm{x} \text {, in any world, is not an F) }
\end{aligned}
$$

With such a deduction as $L u a$, the other forms of its square undermine credence being a function. Given none can be a truth which supersedes being, each form can have rational truth-values $X$ of $C D$ for hypothetical 
proportions; the concepts of likelihood and the reality of their world contradicts the one-way capacity of probabilities for the other worlds! Statistical inference for counterparts implies any imagined correspondence which comes after their correlation, so a superseding truth is being presupposed after all; Lewis' worlds contradict themselves.

Scientific theories that supersede other theories cannot be recognized as following from sound inference if their experimental evidence can only ever be in a correlation to their being. Demonstrating doppelga"ngers or even identical twins that do not have unique counterparts in any world is pointless if Lewis' credence function is inadmissible for Lua even though it depends on it.

Lewis' big bad bug (1994) is not a theory of chance, as if one theory cannot be superseded by another, but the failure of his cherished Principal Principle to hold for the dialectic. Neither does its reasoning which is only a reckoning (Hegel 1832, 32) demonstrate that natural language understanding of unscientific concepts could have existed without the history of philosophy ${ }^{7}$ for which the understanding of modality has played a major part. Only by considering what is in opposition to the theory as well as containing it within a higher standpoint can it be superseded. Demonstrating it is otherwise would mean there can be no dialectic. This should be obvious, but it is all too easy to be ignorant of the one-way capacity of inference, especially when it is taken to be a norm. With one-sided inferences, it is easy to assert the possibility of a non-modal logic even though it isn't true for the actuality of inferential relations. Such an understanding of belief merely presupposes that the structural relations of inferences are undermined by the chance of their being. This should not count as evidence that they do not exist within the dialectic or fail to comprehend it.

By satisfying the concepts of correspondence and distribution, the relation of the likelihoods can have an identity within a manifold that is truth preserving. ${ }^{8}$

Subjectivity cannot be reduced to contingency even if it is assumed to be otherwise. Doing so would make all reasoning equal, which is an absurd and dangerous notion often used to undermine scientific evidence. Even without refutation, it can easily be affirmed that inferences can only be sound given they are modally significant, e.g., learning a language of mathematics to do mathematics. Else the analysis of their functional forms as a property of their being must be denied.

Once the rationality of the inference's capacity is introduced, the empirical presupposition is; the illusion of the $C D$ is transcended by the formalization of its relations. Predication of two-sided and one-sided inferences on their own is merely the belief or experience of their difference. Whereas within a square of opposition, empirical thinking is indifferent to their relation as it would deny any belief which can have a better understanding of their concept; as they are merely facts resembling experiences derived from the copying of ideas.

Reasons for their interpretation establish the facts of the understanding which are also experienced, which tell us how well they correspond to real experiences. Of course, the empiricist doesn't see that their understanding of concepts requires an understanding of experiences not separated from them even though it presupposes there is a separation; its theoretic position comes first. To get around this, knowledge for the propositional forms of inference is reduced to the truth values that correspond to the hypothetical proportions of probability theory. These merely correlate with that which can be verified for experience and so leave an analysis of the theory itself being dependent on belief and not on what is true for and false for the actuality of experience. Beliefs necessarily true make experience contingent. 
Formalists do not just take the correlation of the probabilities with experience to be true, but rather take the theory as it is for-itself, being a belief of beliefs. Empiricists and even realists being skeptical of such a meta-belief would deny the theory which constitutes any knowledge, as this is given by experience in-itself. This leaves the empiricist with the problem of other understandings that are presupposed and not in a relation to experience where there is no credence; formal thinking is taken to be dependent on empirical thinking when in fact the opposite is true. Taking the matter of truth further, the speculative thinker can claim it is what is necessary, possible, or contingent for the relation of inferences that determines the theories validity. Further still by adding intuition to speculative thinking, the proof of the later relations as well as what is impossible for them provides a reason for an even higher standpoint of system.

If reality is just a possibility rather than determined by the facts of experience, the confusion is further exacerbated for empirical, realist, and formal thinking by the likelihoods of Bayes' theorem. Lewis' (1980; 1994) Principal Principle depends on it. Proportions are not considered just for hypothetical reasons as if detached from what is possible for speculative reason. Prior belief is determined empirically, not by the knowledge of the properties probabilities that can be selected as a possibility from all the other properties, but only the independent ones. Now if the truth of the matter only comes after or supervenes being (Lewis 1994, 474), being is confirmed by the facts, so this understanding of truth cannot have any truth before its being. Prior belief is being presupposed to be contingent to begin with. It therefore contradicts itself by being absolute, so hypothetical proportion can be asserted to correlate to predicted distributions. Nothing improbable can be deduced from its function. Being in a probable or improbable relation cannot be demonstrated deductively. Since proportions so conceived have a speculative value with a hypothetical one, it is understandable that their theoretical value need not be scientific or mysterious. This being so, the credence in a correlation does not double up as the credence in a correspondence.

This I take it means hypothetical proportion within a distribution is superseded by the chance of their relation which can be other than an abstract possibility. If I am right, with abstract possibility, there can be no confidence that its understanding for the calculation of chances constitutes a scientific method.

Taking the likelihood to be the reason for events that can be independent or dependent, it can be inferred that there can be more or less confidence in its concept. This is not so with taking the probability function with a fixed hypothesis to be what is most likely for some set of hypotheses, as its calculations not only presuppose the way a property is distributed but also undermines their interaction with other properties. Why a probability should be determined by one of many possible orderings is not explained.

Naming the identity a tautology (Ha'jek 2003; Fitelson and Ha'jek 2014); taking Pfeifer's argument for one: $\operatorname{pr}(T \mid A)=1$ and $\operatorname{pr}(\perp \mid A)=0$ and altering them to get $\operatorname{pr}(T \mid B)=1$ and $\operatorname{pr}(\perp \mid B)=0$, so you can have intermediate values: $\operatorname{pr}(A \mid B)$; the antecedent need not correspond to the consequent or both count as conclusive evidence that the probability has predicted whether the proportion corresponds to a distribution or not. It is too easy to claim an intuitionist position (Weatherson 2003; Roeper \& Leblanc 1999) while working within a formalist paradigm. Given a distribution of properties can be predicted, the identity, difference, and negation axiom do not need to contradict each other; consider how a square of modality could be deduced:

$$
\begin{aligned}
& \square \operatorname{pr}(T \mid A \wedge B), \\
& \neg \nabla \operatorname{pr}(T \mid A \vee B), \\
& \diamond \operatorname{pr}(T \mid A \vee B)
\end{aligned}
$$




$$
\nabla \operatorname{pr}(T \mid A \rightarrow B),
$$

given the later implies $\nabla \operatorname{pr}(T \mid \neg A \wedge B)$, so it could also be deduced that $\neg \nabla \operatorname{pr}(T \mid A)$ is valid for impossibility; are the absurdities $\perp$ just supposed to be those squares which cannot be arranged for a theory of probability? Whatever is postulated the contradictions remain as it does the correlations of probabilities for the proportions of a distribution.

The probabilistic justification of inference for the of justifying the negation axiom: $\neg \operatorname{pr}(A \mid B)=1-\operatorname{pr}(A \mid B)$ is also a problem, as it leaves the theory with a negation that is still a probability for the same proportion which is no longer independent being in opposition to it.

This is a very important but subtle point as what is negated still has a chance of correctly predicting the same proportion. E.g., by this interpretation for the binomial with a proportion $p=1 / 2$, the prediction of one unit out of three states can be split between $3 / 8$ and $5 / 8$, so the difference in terms of making a successful or unsuccessful prediction can be measured. Likewise, the states for $p=1 / 3$ and $p=2 / 3$ as well as any other proportion for the ranking of discrete or continuous functions. Truth preservation only works for $\mathrm{pr} \mid \mathrm{im}(\mathrm{p} / \mathrm{d})$, as the likelihood of each $p$ is negated by just presupposing there is no difference; $\neg p r \mid i m(p / d)$ simply means $[u$, $1 / d-(d-1) / d]$ where I take $u$ to be when their difference is unknown and $d$ is the distribution of difference for any set of states, which is not the case. No confidence can be had even when the successes are high or low if negation is truly to mean the function is negated. Taking unity away from difference negates the truth of it.

Explaining why the formal understanding isn't coherent with an intuitive and speculative one, reasons need to be provided that go beyond the obvious numerical substitutions which make its concept of unity false for impossibility. Pfeifer (2014) claims he can be certain that the population of the Vatican City is less than that of the United States, but this is not supposed to be a tautology. Despite this the assertion of $\operatorname{pr}(T \mid A)=1$ also presupposes an existential relation, no necessity can be derived from a unit of being other than reducing all certainties to existence! What is more, given $T$ is self-referential, the tautology is contingent as well. Universality is therefore reduced to the certainty being a self-subsistent set; given the properties of the thing constitute its substance, its self-subsistent matter is disconnected from its improbability.

How well probability functions work for making predictions is usually analyzed by narrowly focusing on definitions as they relate to games of chance. Being limited to the difference of independent variables, they cannot be effective at predicting how well their proportions correspond to their given distributions, so stochastic analysis can be further challenged. Calling the function that is sound for making inferences about chances a likelihood function, it seems obvious that it is in a logical relation to a probability function. This gives the dialectician a reason for taking one understanding of logic to include, but not be held captive to an understanding belonging to a lower standpoint of system. Consider games of chance that can be played with fixed and alternating proportions; imagine one where unknown binary rules are used e.g., write only those decimals in succession according to the binary rule where they are in correspondence with odd and even units, or odd and even units with even zero's; from $\{1,11,100,111,1001,1010,1100,1111 \ldots\}$ for $\ldots 2^{3}, 2^{2}, 2^{1}, 2^{0}$, as you write the units or zeros from left to right, the decimals you get $\{1,3,4,7,9,10,12,15 \ldots\}$, which could correspond to a binary sequences in modular $2:\{1,0,0,1,0,0,0,1 \ldots\}$.

\subsection{Probabilities without Being in a Difference to Improbabilities'}

It then seems probability theory is stuck, as any presupposed ordering or curve fitting depends on the assumption of a correlation; no confidence can be had in its predictions and so the derivation of the above rule 
would be impossible. Given the success of the improbabilities, the variables are not independent, so it can be deduced that the units and zeros do not correspond to the combinatorial proportions of binomial probabilities or any other probability function on the supposition of an invariant proportion. Taking the stochastic concept even further so the initial conditions unknown; other rules can be introduced and seeded by a time stamp leaving the probability theory in the dust. What is happening is that from the antecedent relation for the function: $p r \mid i m(p / d)^{9}$ but without a knowledge of it, the postcedent has been deduced: $\operatorname{pr}(p / d)$, so the consequent of adhering to the former is the likelihood, $l p$ where as of the later given $\operatorname{pr}(p / d) /=l p$. With this the logical justification for the predication of the inferences that could verify a belief in probability, theory for the soundness of its inferences is missing.

This can be seen from the structure for the two-sided or two-way selection of antecedents $A k$ and the inclusive disjunction $V i$ for their possible selection $P k$ of their postcedents that are deduced to get the exclusive disjunction $V e$ of their consequences $C k$ for the knowledge $k$ of the properties disposition:

$$
\frac{A_{0} \vee_{i} P_{0} \wedge A_{1} \vee_{i} P_{1} \wedge \ldots \wedge A_{k} \vee_{i} P_{k}}{C_{0} \vee_{e} C_{1} \vee_{e} \ldots \vee_{e} C_{k}}
$$

\section{Definition}

Let $M i$ be a model of inferences where ( $I a, U m, D p, S \Gamma) I a$ stands for the interpretation of the axioms, A5, $U m,{ }^{10}$ the understanding of modality, $D p$, the disposition of the properties, and $S \Gamma$, the formulae of the system using the usual logical and mathematical operations. Consider the language Ia of ISL as a minimal set of definitions to construct different functions for the axioms: +, -, *, !, be an defined set of arithmetical operations; let the logical operations be: |(or and both), $\subset$ (inclusion), $\subseteq$ (proper subset), $V_{i}$ (inclusive or), $\neg$ (negation). Taking $p$ to be the proportion or relative frequency of a property's states, $d$ is their distribution and $t$ is the total of the states being counted.

Also let $s$ be the total for the state being selected; $t$ ! and $s$ ! are the factorial of the states being counted; $C$ is the combinator for

where

$$
{ }^{t} C_{s} p^{s}(1-p){ }^{t-s}
$$

$$
{ }^{t} C_{s}=\frac{t !}{s !(t-s) !} .
$$

Also if $\mathrm{p} / \mathrm{d}$ is the proportion of the states given a distribution of states having a property, $\operatorname{pr} \operatorname{im}(e) \subset s_{0} p_{n}, s_{1} p_{n} \ldots s_{k} p_{n} \ldots s_{n} p_{n}$ and for properties having states, $\operatorname{pr} \mid \operatorname{im}(E) \subset p_{0} s_{n}, p_{1} s_{n} \ldots p_{k} s_{n} \ldots p_{n} s_{n}$ where $E$ is a set of properties; this expands the right side of the conditional for the two-sided inference.

A1. $p_{h} \in d_{p}$ that is the hypothetical proportion belongs to the distribution of the properties' states iff the distribution of possible worlds implies the possible worlds are not a proper subset of the real worlds: $P_{d} \supset w_{p} \nsubseteq w_{r}$;

A2. $\neg p r|i m(p / d)=1-p r| i m(p / d)$ 
A3. $\operatorname{pr} \mid \operatorname{im}(p / d) \in l_{p}$ the difference between the probabilities and the improbabilities belongs to the likelihood of the proportion

A4. $p r(p / d) \neq l_{p}$

A5.

$$
\begin{aligned}
& l_{p}= \begin{cases}\operatorname{pr}(e) & \text { when property's successes } \sum_{p r}^{s} p>\sum_{i m}^{s} p, \\
\operatorname{im}(e) & \text { when property's successes } \sum_{i m}^{s} p>\sum_{p r}^{s} p .\end{cases} \\
& L_{P}= \begin{cases}\operatorname{pr}(E) & \text { when properties successes } \sum_{p r}^{p} s>\sum_{i m}^{p} s, \\
\operatorname{im}(E) & \text { when properties successes } \sum_{i m}^{p} s>\sum_{p r}^{p} s .\end{cases}
\end{aligned}
$$

if $\operatorname{pr}(p / d)>\operatorname{im}(p / d)$ then $\operatorname{pr}(p / d) \supset d_{p}$ or if $i m(p / d)>\operatorname{pr}(p / d)$ then $i m(p / d) \supset$ $d_{p} \operatorname{clsc} \operatorname{pr}(p / d)=\operatorname{im}(p / d)$

$D_{p}$ includes real worlds $w_{r}$ and possible worlds $w_{p}$; their hypothetical proportions $p_{h}$ and particular distribution of their properties $d_{p}$; implies the possible worlds are not a proper subset of the real ones. Given $\left(\forall w_{p} \wedge \forall w_{r}\right) \in S_{\Gamma}$, $\left(\forall w_{p} \subseteq \forall w_{r}\right) \notin S_{\Gamma}$

Proposing a counterexample that also holds for other functions, be they discrete or continuous cannot but confound the independent ordering of the states from their dependent ordering. E.g., if $p=1 / 2$ and the probability of getting $0,0,1$ or $0,1,0$ or $1,0,0$ is $3 / 8$, whereas for $5 / 8$ is the improbability of getting them, not that of getting $0,0,0$ or $0,1,1$ or $1,0,1$ or $1,1,0$ or $1,1,1$. Taking what is presupposed to be uniform by limiting it to independent selections for the binomial, the conditions of the probability function do not satisfy what can be predicted for this uniformity as the difference from it and what is improbable for it can be calculated; so A3 is valid; the negation of difference should be no difference! Natural orderings for the probable or improbable correspondence of proportions and the distributions of properties therefore undermines counterpart worlds, so A1 is true.

The reason for this is that they require the presupposition of $\operatorname{pr}(p / d)$ for the justification of a correlation to possible worlds. This contradicts the worlds having an identity according to the temporal and spatial laws of actual worlds.

Actual worlds cannot have the same properties at the same time in their relation to other properties. Aristotle's Metaphysical principle is therefore still applicable as deduced from the concept of rational inference. It is in intuitive opposition to the contingency of empirical thinking where possibility is an existential reality. Due to the capacity and potential of inferences being in an opposition, their unity cannot be in an actual relation of contingency for possibility and necessity. The duality of inferences as a disunity would merely suggest their one and two-way is a structure reducible to their likelihood.

\section{Conclusion}

That is, it can be argued that there is no perfect representation of symbolic language, and is not an adequate reason to deny the possibility of a sound system of formula for processing uncertain information. Neither is taking one symbolic language, such as that of the Principia Mathematica to be an adequate reason to deny the modal truths of mathematics, given any translation of its corpus within a core Um, where Um 
represents the different understanding of modality, where speculative thinking can be distinguished from the limitations of formal and empirical thinking given the theorems of ISL hold. Most obvious is the limitation of excluded-middle for constructing inferences that are in a relation. It is simply a case of the antecedent being true or false for the postcedent, so their consequences can be integrated. To do this, take modality to be a justified condition that satisfies 4 valid theorems given the arguments for $p r \mid i m$ avoids the error of the exclusive disjunction $\operatorname{pr}(e, E)$ Ve im $(e, E)$ for one-sided inferences.

Introducing the modalities necessity; $\square$, possibility; $\diamond$, impossibility; $\neg \diamond$ and contingency; $\nabla$ and their relations; $=,>, \geq$ :

Theorem $1 \square \forall(p r \mid i m) \forall(p r) \forall(E)(p r \mid i m(E)>p r(E))$

Theorem $2 \diamond \exists(p r \mid i m) \exists(p r) \forall(E)(p r \mid i m(E) \geq p r(E))$

Theorem $3 \neg \oslash \exists(p r \mid i m) \exists(p r) \forall(E)(p r \mid i m(E) \geq p r(E))$

Theorem $4 \nabla \exists(p r \mid i m) \exists(p r) \exists(E)(p r \mid i m(E)=p r(E))$

Lemma's can then be constructed to falsify false theorems for necessary universal predication i.e., $\mathrm{D} \forall(p r \mid i m) \forall(p r) \forall(E)(p r \mid i m(E)=p r(E))$.

Taking the modal symbols to represent and so simplifying the idea of these theorems, they are synonymous with the predicated propositions you get from figure 1; the diagonals are contradictions and the top horizontal connects the contraries, and the bottom one connects the sub-contraries while the verticals hold for entailment.

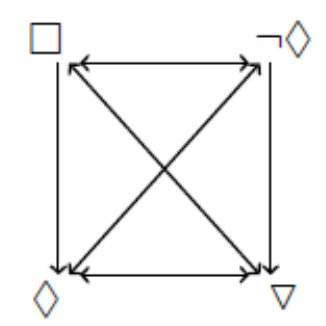

1. A modal square of opposition

$$
\begin{aligned}
& \operatorname{pr}(Y x / X x)=1 \quad \operatorname{pr}(Y x / X x) \neq 1 \\
& \stackrel{\operatorname{pr}(\neg Y x}{\longleftrightarrow} / X x)>0
\end{aligned}
$$

2. A probabilistic relation of non-opposition

Corollary: given the $M f-M$, where $M$ is a manifold and $M$ is a representation of what it seems, it is reasonable to differentiate $I S L$ from Speculative Logic $S L$, Formal Logic $F L$, and Empirical Logic $E L$ if and only if the understandings of logic have a modal condition which defines their semantics unconditionally. In fact, it seems that they cannot do otherwise for their Credence $C$ which is included within the function of the dialectic belonging to a speculate corpus:

$$
D(C \mid M)= \begin{cases}I S L, & \text { if } \square, \diamond, \neg \nabla, \nabla ; \\ S L, & \text { if } \square, \nabla, \nabla ; \\ F L, & \text { if } \neg \square, \nabla, \nabla ; \\ E L, & \text { if } \neg \square, \neg \diamond, \nabla .\end{cases}
$$


Lemma: $\nabla \forall(p r \mid i m) \forall(p r) \forall(E)(p r \mid i m(E)=p r(E))$ as an hypothesis, it is false by Theorem 4; no amount of rigorous testing for errors is going to prove it otherwise so $\neg \models \nabla \forall(p r \mid i m) \forall(p r) \forall(E)(p r \mid i m(E)=p r(E))$. As if it were all, the propositions holding for the theory of probability would be in correspondence with their possible distributions. Since they are not, the contradictories of the square are the contradictories belonging to different understandings of modality!

Only when $E L$ or any other understanding of logic is presupposed does it appear that the square of opposition is otherwise; the structure of the inferences for $I S L, S L$, and $F L$ seems sound in that their comprehension is reasonable in a way that they are not for figure 2; within the dialectic, there is no way to put the brakes on the philosophy logic and science just for the sake of agreement. Unlike the hypothetical corpus, the principle of the dialectic hereof interpreted means there is a correspondence between the understandings of the concepts and the actuality of their experience. This also takes Hegel's (1807, 152; 1832, 205) Phenomenology for the Science of System and Speculative Logic (Hegel 1832, §9) into consideration; given his criticism of probability in regard to the truth of the matter, it shouldn't be overlooked. Neither does Hegel deny that the dialectic of chance can be developed so as to take account of modality. It is only for the relation of the functions concept resting on the unity of oppositions that impossibility is not well defined. Moreover, using probability theory to justify the categorical forms (Slater 2012,142) doesn't work for a square of opposition as can be seen from figure 2,

$\operatorname{pr}(Y x / X x)=1$ (i.e., all Xs are Ys) is not the contradictory of $\operatorname{pr}(Y x / X x)>0$ (i.e., some Xs are not Ys) as both are true for the interval: $[0,1]$ of probability theory. In fact Slater admits $\operatorname{pr}(Y x / X x) /=1$ (i.e., not all Xs are Ys) does not entail $\operatorname{pr}(Y x / X x)>0$ so neither is $\operatorname{pr}(Y x / X x)>0$ (i.e., some Xs are Ys), the contradictory of $\operatorname{pr}(Y x / X x) /=1$ (i.e., not all Xs are Ys) or the entailment work for the left hand side. This I think provides enough evidence to move beyond Aristotle, Hegel, and the Idealists contribution to the dialectic analysis of inference.

\section{Notes}

1. It is only with the two-way capacity of inference that experiences can be intelligible. With only a one-way capacity, they are not. For this reason, unlike Makin and Denyer (2000), I do not think it is valid to presuppose that a genuine two-way capacity corresponds to a doctor's medical skill; a pseudo two-way capacity corresponds to a magnet and a one-way capacity as that of fire to produce heat etc. Like anybody else, doctors can make use of rational or irrational inferences in their diagnosis; the capacities of inferences are not the capacities of experiences, but of the way they are processed as thoughts. Not being intelligible, thoughts as objects would be merely in themselves and not in a self-relation for-themselves, or to that which is other-than-themselves, to experiences.

2. Sobor (1988) equates proportions with probabilities, but chances themselves can be calculated in many different ways while proportions are primarily the quantitative attributes of properties.

3. Quentin Meillassoux's $(2006,227)$ critique of correlationism is certainly problematic for probability theory; if thinking merely correlates to being the value of the inference becomes uncertain, deduction has no speculative value. Meillassoux gives the impression that he is abducting probability theory to attack Hegel's dialectic. This is easily refuted and also supported by his critique of probable in regard to the truth; no principle can be demonstrated by being probable. There is therefore simply a failure to realize that inferences do contradict themselves if their relations can be universal and or existential. Just being affirmed or asserted and denied or refuted takes the logic out of science, for what can be verified or falsified.

4. This selection is not to belittle their contributions, or intended to be a definite list of all those who have worked or are working on developing the logic of inference and chance. Rather it is to help the philosophical reader understand that none of their expositions have resolved all the conceptual problems, or prevent them being developed further.

5. Other attempts (Taper \& Lele 2011) to justify a likelihood principle being sound for prior probabilities fail to take into account Hegel $(1807$; 1832) and Ayer's (1957) obvious point that statements of probabilities cannot be logically true for their relation if they are just probable. Clearly the notion that proof is probable or likely for the demonstration of theorems is absurd. 
6. If I am right, there is more to the BKH interpretation (Kolmogorov 1932), namely because probabilistic interpretations of Intuitionism (Moran \& Leblanc 1983; Roeper \& Leblanc 1999; Weatherson 2003) fail to take into account the modal structure of inferential relations that are in an opposition. A modal square need not rely on the mythical structure of S5 Chihara (1998; 2004); it can be interpreted constructively as D is a proof of necessity, $\downarrow$ is a proof of possibility, $\neg \downarrow$ is a proof of impossibility, and V is a proof of contingency.

7. Given the success of all the conferences on the Square of Opposition, it could be called an awakening for those who ignore all the hard work done by the Greeks and later those of the enlightenment; Hegel (1832) makes his intentions about his mission plain enough; the Science of Logic is not intended to ground the concept of logic, or to justify the advance of its scientific method, "but rather to make more intuitable, by means of some explanations and reflections of an argumentative and historical nature, the standpoint from which this science ought to be considered," 23; by containing the unity of itself and its opposite, a system of concepts can be developed that contains nothing extraneous, 33 .

8. What is valid for necessity, possibility, impossibility, contingency, and reality shouldn't be confounded by speculating about what is real for actuality, so reducing the truth of the matter to mere appearance or the utility that conforms to the sensibilities of an imagined being, i.e., just because the dinosaurs were real, does mean their counterparts still exist on other worlds.

9. Using the stoke function allows for the third value where $\mathrm{pr}$ and $\mathrm{im}$ are equal.

10. The subscripts represent the species for general class symbols, but can be used to refer to particular ones as well, i.e., Uem would be an understanding of empirical modality.

\section{Works Cited}

Ackermann, Robert. "Inductive Simplicity.” Philosophy of Science 28.2 (1961): 152-60. Probability, Confirmation, and Simplicity. Ed. Marguerite Foster \& Michael Martin. New York: The Odyssey Press Inc., 1966. 322-31.

Ayer, Alfred. "Observation and Interpretation." Ed. S. Ko“ner. London: Butter-Worths Scientific Publications, 1957. 12-17. Probability, Confirmation, and Simplicity. Ed. Marguerite Foster \& Michael Martin. New York: The Odyssey Press Inc., 1966. 67-73.

Barker, Stephen. "Induction and Hypothesis." Ithaca: Cornell University Press, 1957. 82-90. Probability, Confirmation, and Simplicity. Ed. Marguerite Foster \& Michael Martin. New York: The Odyssey Press Inc., 1966. 61-73.

---. "On Simplicity in Empirical Hypotheses, Philosophy of Science." 28.2 (1961): 162-71. Probability, Confirmation, and Simplicity. Ed. Marguerite Foster \& Michael Martin. New York: The Odyssey Press Inc., 1966. 240-9.

Be'ziau, Jean-Yves and Jacquette Dale. Ed. Jean Yves-Be'ziau and Dale Jacquette. Around and Beyond the Square of Opposition. Springer Basel, Heidleberg: Birkha“user, 2012.

Bohm, David. "The Special Theory of Relativity." 1996. Routledge Braithwaite, Richard, B. "Scientific Explanation.” New York: Cambridge University Press, 1959. 264-91. Probability, Confirmation, and Simplicity. Ed. Marguerite Foster \& Michael Martin. New York: The Odyssey Press Inc., 1966. 343-63.

Carnap, Rudolf. “On Inductive Logic.” Philosophy of Science 12.2 (1945): 72-97. Probability, Confirmation, and Simplicity. Ed. Marguerite Foster \& Michael Martin. New York: The Odyssey Press Inc., 1966. 35-61.

Chihara, Charles. The Worlds of Possibility (Modal Realism and the Semantics of Modal Logic). Oxford: Clarendon Press, 1998.

---. A Structural Account of Mathematics. Oxford: Clarendon Press, 2004.

Cohen, Morris \& Nagel. "An Introduction to Logic and Scientific Method." 1962. 267-9, Probability, Confirmation, and Simplicity. Ed. Marguerite Foster \& Michael Martin. New York: The Odyssey Press Inc., 1966. 374-5

Fitelson, Branden \& Ha'jek Alan. "Declarations of Independence.” Synthese, 2014. 1-17.

Glass, David and McCartney Mark. "Explanatory Inference under Uncertainty." Lecture Notes in Computer Science 8669. Salamanca, Spain: Springer, 2014. 215-22.

Grossman, Jason. "The Likelihood Principle." Handbook of the Philosophy of Science, Vol. 7: Philosophy of Statistics. Ed. Prasanta Bandyopadhyay and Malcolm Forster. Series Editor: Dov Gabbay, Paul Thagard, and John Woods. Oxford: Elsevier B.V., 2011. 553-80.

Ha'jek, Alan. "What Conditional Probability could not Be." Synthese 137 (2003): 273-323.

Hall, Ned. "Two Mistakes about Chance." Australasian Journal of Philosophy 82.1 (2004): 93-111.

Hegel, Georg Wilhelm Friedrich. Phenomenology of Spirit. Trans. A. V. Miller. Oxford: Oxford University Press, 1977.

---. Science of Logic. 1813 book two of volume one, 1816 volume two and book one revised 1832. Trans. \& Ed. George di Giovanni. New York: Cambridge University Press, 2010. 
---. The Encyclopaedia Logic. Trans. T. F. Ger-aets, W. A. Suchting, and H. S. Harris. Indianapolis/Cambridge: Hackett Publishing Company, Inc., 1991.

Hillman, Donald. "The Measurement of Simplicity." Philosophy of Science 29.3 (1962): 225-52. Probability, Confirmation, and Simplicity. Ed. Marguerite Foster \& Michael Martin. New York: The Odyssey Press Inc., 1966. 253-79.

Joyce, James. "A Defense of Imprecise Credences in Inference and Decision Making." Philosophical Perspectives, 24. Epistemology. 2010. 281-323.

Kimbrough, Steven Orla. "On the Use of Likelihood as a Guide to Truth." PSA: Proceedings of the Biennial Meeting of the Philosophy of Science Association, Vol. 1. Collected Papers. Chicago: University of Chicago Press, 1980. 117-28.

Kolmogorov, Andrei. "Zur Deutung der intuitionistischen logik, Math-ematische Zeitschrift.” 35, 58-65. Trans. Paolo Mancosu. Oxford: Oxford University Press, 1998. 328-34.

---. Foundations of the Theory of Probability (2 English Ed.). New York: Chelsea Publishing Company, 1956.

Kyberg, Jr. Henry. Science \& Reason. Oxford: Oxford University Press, 1990.

Lenz, John. "Carnap on Defining 'Degree of Confirmation.” Philosophy of Science 23.3 (1956): 230-6. Probability, Confirmation, and Simplicity. Ed. Marguerite Foster \& Michael Martin. New York: The Odyssey Press Inc., 1966. 73-79.

Lewis, David. "Counterpart Theory and Quantified Modal Logic.” The Journal of Philosophy, Vol. LXV, No. 5, 1968. 113-26. “A Subjectivists Guide to Objective Chance." Studies in Inductive Logic and Probability, Vol. II. Ed. Richard Jeffrey. Berkeley: University of California, 1980. "Humean Supervenience Debugged." Mind 103.412 (1994): 473-90.

Makin, Stephen \& Denyer Nicholas. “Aristotle on Modality.” Proceedings of the Aristotelian Society 74 (2000): 143-61.

Meillassoux, Quentin. "Potentiality and Virtuality." The Speculative Turn. Trans. Robin Mackay. 2011. Ed. Levi Bryant, Nick Srnicek, and Graham Harman. Victoria: Re-press, 2006, 224-36.

Morgan, Charles and Leblanc Hugues. "Probabilistic Semantics for Intuitionistic Logic." Notre Dame Journal of Formal Logic 24.2 (1983): 161-80.

---. "Probability Theory, Intuitionism, Semantics, and the Dutch Book Argument." Notre Dame Journal of Formal Logic 24.3 (1983): 289-304.

Pfeifer, Niki. "Reasoning about Uncertain Conditionals." Studia Logica 102 (2014): 849-66.

Popper, Karl. Logic of Scientific Discovery. London: Routledge Press, 1959.

Reichenbach, Hans. "Theory of Probability.” Berkeley: University of California Press, 1949. 68-69; 372-83; 434-42; 470-82. Probability, Confirmation, and Simplicity. Ed. Marguerite Foster \& Michael Martin. New York: The Odyssey Press Inc., 1966. 423-34.

Roeper, Peter and Leblanc Hughes. "Absolute Probability Functions for Intuitionistic Propositional Logic." Journal of Philosophical Logic 28 (1999): 223-34.

Russell, Bertrand. The Principles of Mathematics. 2nd Ed. London: W W Norton \& Company, 1969.

---. An Inquiry into Truth and Meaning. London: George Allen and Unwin Ltd., 1940.

---. "Human Knowledge, Its Scope and Its Limits." New York: Simon \& Schuster, 1962. 487-96. Probability, Confirmation, and Simplicity. Ed. Marguerite Foster \& Michael Martin. New York: The Odyssey Press Inc., 1966. 376-83.

Slater, Hartley. "Back to Aristotle." Logic and Logical Philosophy 20 (2011): 257-83.

---. "The Right Square." Ed. Jean Yves-Be'ziau and Dale Jacquette. Around and Beyond the Square of Opposition. Springer Basel, Heidleberg: Birkha"user, 2012.

Sober, Elliott. "Likelihood and Convergence." Philosophy of Science 55.2 (1988): 228-137.

Taper, Mark and Lele Subhash. "Evidence, Evidence Functions, and Error Probabilities." Handbook of the Philosophy of Science Vol. 7: Philosophy of Statistics. Ed. Prasanta Bandyopadhyay and Malcolm Forster. Series Editor: Dov Gabbay, Paul Thagard, and John Woods. Oxford: Elsevier B.V., 2011. 513-49.

Van Fraassen, Bas. "Fine-Grained Opinion, Probability, and the Logic of Full Belief." Journal of Philosophical Logic 24.4 (1995): 349-77.

Weatherson, Brian. "From Classical to Intuitionistic Probability." Notre Dame Journal of Formal Logic 44.2 (2003): 111-23. 